$$
\begin{array}{llll}
\lrcorner & \lrcorner
\end{array}
$$

Squareful numbers in hyperplanes

\lrcorner Karl Van Valckenborgh

」

\rfloor

象

」 


\title{
Squareful numbers in hyperplanes
}

\author{
Karl Van Valckenborgh
}

\begin{abstract}
Let $n \geqslant 4$. In this article, we will determine the asymptotic behavior of the size of the set of integral points $\left(a_{0}: \cdots: a_{n}\right)$ on the hyperplane $\sum_{i=0}^{n} X_{i}=0$ in $\mathbb{P}^{n}$ such that $a_{i}$ is squareful (an integer $a$ is called squareful if the exponent of each prime divisor of $a$ is at least two) and $\left|a_{i}\right| \leqslant B$ for each $i \in\{0, \ldots, n\}$, when $B$ goes to infinity. For this, we will use the classical Hardy-Littlewood method. The result obtained supports a possible generalization of the Batyrev-Manin program to Fano orbifolds.
\end{abstract}

\section{Introduction}

The problem we consider can be related to a question Campana posed concerning rational points on orbifolds. A good overview is given for example in [Abramovich 2009; Poonen 2006; Campana 2005]. Examining the orbifold $\left(\mathbb{P}^{1}, \Delta\right)$ with $\mathbb{Q}$ divisor $\Delta=1 / 2 \cdot[0]+1 / 2 \cdot[1]+1 / 2 \cdot[\infty]$, it is explained for example in [Poonen 2006] why it is reasonable to expect that the set

$\left\{\left(a_{1}, a_{2}, a_{3}\right) \in \mathbb{Z}^{3}: a_{1}+a_{2}=a_{3}, a_{1}, a_{2}, a_{3}\right.$ are squareful,

$$
\left.\max \left\{\left|a_{1}\right|,\left|a_{2}\right|,\left|a_{3}\right|\right\} \leqslant B, \operatorname{gcd}\left(a_{1}, a_{2}, a_{3}\right)=1\right\}
$$

will asymptotically behave as $C \cdot B^{1 / 2}$ as $B$ tends to infinity.

Since this question turns out to be too difficult at the moment, we generalize to a higher-dimensional analogue $\left(\mathbb{P}^{n-1}, \Delta\right)$, where now $\Delta$ is the $\mathbb{Q}$-divisor $\Delta=1 / 2 \cdot\left[H_{0}\right]+\cdots+1 / 2 \cdot\left[H_{n}\right]$ with $H_{i}$ the hyperplane defined by $X_{i}=0$ for $i \in\{0, \ldots, n-1\}$ and $H_{n}$ defined by $X_{0}+\cdots+X_{n-1}=0$. In analogy with the one-dimensional case, a point $P=\left(a_{0}: \cdots: a_{n-1}\right) \in \mathbb{P}^{n-1}(\mathbb{Q})$ (we assume $a_{0}, \ldots, a_{n-1} \in \mathbb{Z}$ and $\left.\operatorname{gcd}\left(a_{0}, \ldots, a_{n-1}\right)=1\right)$ will be called a rational point in Campana's sense on $\left(\mathbb{P}^{n-1}, \Delta\right)$ if for every $i \in\{0, \ldots, n\}$ and every prime $p$ for which the reduction of $P$ is contained in the reduction of $H_{i}$ modulo $p$, we have $i_{p}\left(P, H_{i}\right) \geqslant 2$, where $i_{p}\left(P, H_{i}\right)$ denotes the intersection number of $P$ and $H_{i}$ above the prime $p$. These conditions will be satisfied if $a_{i}$ is squareful for every

MSC2010: primary 11D45; secondary 14G05, 11D72, 11P55.

Keywords: squareful, Campana, asymptotic behavior. 
$i \in\{0, \ldots, n-1\}$ and if $\sum_{i=0}^{n-1} a_{i}$ is also squareful. We denote the set of all such rational points by $\left(\mathbb{P}^{n-1}, \Delta\right)(\mathbb{Q})$. Using the height function

$$
H\left(x_{0}: \cdots: x_{n-1}\right)=\max \left\{\left|x_{0}\right|, \ldots,\left|x_{n-1}\right|,\left|\sum_{i=0}^{n-1} x_{i}\right|\right\}
$$

the set of points $P \in\left(\mathbb{P}^{n-1}, \Delta\right)(\mathbb{Q})$ of bounded height is denoted $\left(\mathbb{P}^{n-1}, \Delta\right)(\mathbb{Q}) \leqslant B$.

Defining the canonical divisor of the orbifold $\left(\mathbb{P}^{n-1}, \Delta\right)$ as

$$
K_{(\mathbb{P} n-1, \Delta)}=K_{\mathbb{P} n-1}+\Delta,
$$

we have $K_{\left(\mathbb{P}^{n-1}, \Delta\right)} \sim(-(n-1) / 2) \cdot H$ in $\operatorname{Pic}\left(\mathbb{P}^{n-1}\right)_{\mathbb{Q}}$, where $H$ is the hyperplane class of $\mathbb{P}^{n-1}$. Since the height function we use is associated to $H$, a very naive generalization of Manin's conjecture would predict that $\#\left(\mathbb{P}^{n-1}, \Delta\right)(\mathbb{Q})_{\leqslant B} \sim C \cdot B^{(n-1) / 2}$ for some constant $C>0$, as $B$ tends to infinity. Our main goal is to prove the following theorem.

Theorem 1.1. For $n \geqslant 4$, there exists $a \delta>0$ so that

$$
\#\left(\mathbb{P}^{n-1}, \Delta\right)(\mathbb{Q})_{\leqslant B}=C \cdot B^{(n-1) / 2}+O\left(B^{(n-1) / 2-\delta}\right)
$$

for some constant $C>0$.

In Section 5 we will give an explicit description of the constant $C$ and examine the distribution of rational points on the orbifold $\left(\mathbb{P}^{n-1}, \Delta\right)$.

\section{Description of the proof}

Throughout the article, we will use the following notation.

We will denote the $(n+1)$-tuple $\left(x_{0}, \ldots, x_{n}\right) \in A^{n+1}$ for any ring $A$ by $\underline{x}$. For the nonzero integers we use the notation $\mathbb{Z}_{0}$, that is $\mathbb{Z}_{0}=\mathbb{Z} \backslash\{0\}$. If there exists a constant $C>0$ such that $|f(x)| \leqslant C g(x)$ for real-valued functions $f$ and $g$ with $g$ only taking positive values, we write $f(x) \ll g(x)$ or $f(x)=O(g(x))$. If $C$ depends on other parameters, this will be denoted explicitly when this dependence is important for the computations. We will write $f(x) \sim g(x)$ if $f(x) / g(x)$ tends to one if $x$ goes to infinity. Also, we allow the small positive constant $\varepsilon$ to take different values at different points of the arguments. Finally, for any $\alpha \in \mathbb{R}$ we will write $e(\alpha)=\exp (2 \pi i \alpha)$.

To prove Theorem 1.1, we first restrict ourselves to the set of points

$$
\left(a_{0}: \cdots: a_{n-1}\right) \in\left(\mathbb{P}^{n-1}, \Delta\right)(\mathbb{Q})
$$

for which $a_{i} \neq 0$ for each $i \in\{0, \ldots, n-1\}$ and $\sum_{i=0}^{n-1} a_{i} \neq 0$. We denote this subset by $\left(\mathbb{P}^{n-1}, \Delta\right)(\mathbb{Q})^{+}$. Also, $\left(\mathbb{P}^{n-1}, \Delta\right)(\mathbb{Q})_{\leqslant B}^{+}$indicates the intersection of $\left(\mathbb{P}^{n-1}, \Delta\right)(\mathbb{Q})^{+}$with $\left(\mathbb{P}^{n-1}, \Delta\right)(\mathbb{Q})_{\leqslant B}$.

By the definition of $\left(\mathbb{P}^{n-1}, \Delta\right)(\mathbb{Q})$, we can identify $\left(\mathbb{P}^{n-1}, \Delta\right)(\mathbb{Q})_{\leqslant B}^{+}$with the 
set

$\left\{\left(a_{0}: \ldots: a_{n}\right) \in H(\mathbb{Q}): a_{i} \in \mathbb{Z}_{0}, a_{i}\right.$ is squareful, $\left.\operatorname{gcd}\left(a_{0}, \ldots, a_{n}\right)=1, \max _{0 \leqslant i \leqslant n}\left|a_{i}\right| \leqslant B\right\}$, where $H \subset \mathbb{P}^{n}$ is the hyperplane defined by $X_{0}+\cdots+X_{n}=0$.

Since a squareful integer can be written uniquely (up to the sign of $x$ ) as $x^{2} y^{3}$, where $y$ is squarefree, the latter set in turn corresponds to $\left\{\left(x_{0}^{2} y_{0}^{3}: \cdots: x_{n}^{2} y_{n}^{3}\right) \in H(\mathbb{Q}): x_{i}, y_{i} \in \mathbb{Z}_{0}\right.$ and $y_{i}$ is squarefree,

$$
\left.\operatorname{gcd}\left(x_{0} y_{0}, \ldots, x_{n} y_{n}\right)=1, \max _{0 \leqslant i \leqslant n}\left|x_{i}^{2} y_{i}^{3}\right| \leqslant B\right\} \text {. }
$$

Definition. We define $M(B)$ as the set

$$
\begin{aligned}
\left\{(\underline{x}, \underline{y}) \in \mathbb{Z}_{0}^{2 n+2}: \sum_{i=0}^{n} x_{i}^{2} y_{i}^{3}=0,\right. & \operatorname{gcd}\left(x_{0} y_{0}, \ldots, x_{n} y_{n}\right)=1, \\
& \left.\max _{0 \leqslant i \leqslant n}\left|x_{i}^{2} y_{i}^{3}\right| \leqslant B, \prod_{i=0}^{n} \mu^{2}\left(\left|y_{i}\right|\right)=1\right\} .
\end{aligned}
$$

(Note that for any integer $y \in \mathbb{Z}$, the condition $\mu^{2}\left(\left|y_{i}\right|\right)=1$ means that $y_{i}$ is squarefree.) Also, we denote by $M_{\underline{a}, t}(B)$ the set

$$
\left\{(\underline{x}, \underline{y}) \in \mathbb{Z}_{0}^{2 n+2}: \sum_{i=0}^{n} a_{i} x_{i}^{2} y_{i}^{3}=t, \max _{0 \leqslant i \leqslant n}\left|a_{i} x_{i}^{2} y_{i}^{3}\right| \leqslant B, \prod_{i=0}^{n} \mu_{i}^{\prime}\left(y_{i}\right)=1\right\},
$$

where $a_{0}, \ldots, a_{n}, t \in \mathbb{Z}$ are fixed, $\operatorname{gcd}\left(a_{0}, \ldots, a_{n}\right)=1$ and $\prod_{i=0}^{n} a_{i} \neq 0$. Here, $\mu_{i}^{\prime}$ denotes an arbitrary function $\mathbb{Z}_{0} \rightarrow\{0,1\}$, for each $i \in\{0, \ldots, n\}$.

As a first step in the proof, we will use the classical Hardy-Littlewood circle method to determine an expression for the cardinality of the set $M_{\underline{a}, t}(B)$. Notice that in the definition of $M_{\underline{a}, t}(B)$, we replaced the function $\mu^{2}(\cdot)$ in the definition of $M(B)$ with the more general function $\mu_{i}^{\prime}(\cdot)$. We shall see that applying the circle method is independent of this condition, but nevertheless necessary to derive an asymptotic formula for $\# M(B)$ since squarefree conditions on multiples of the $y_{i}$ will appear as we will explain below. We see that $M(B)$ is a subset of $M_{(1, \ldots, 1), 0}(B)$ (if we take $\mu_{i}^{\prime}(\cdot)$ to be $\mu^{2}(\cdot)$ for each $i$ ), with the additional $\operatorname{gcd}$ condition $\operatorname{gcd}\left(x_{0} y_{0}, \ldots, x_{n} y_{n}\right)=1$ on the solutions. We will take this gcd condition into account using an adapted version of the Möbius inversion.

Identifying $\left(\mathbb{P}^{n-1}, \Delta\right)(\mathbb{Q})_{\leqslant B}^{+}$with $(1)$, it readily follows that

$$
\#\left(\mathbb{P}^{n-1}, \Delta\right)(\mathbb{Q})_{\leqslant B}^{+}=\frac{1}{2^{n+2}} \# M(B),
$$

which implies that an asymptotic formula for $\# M(B)$ induces an asymptotic formula for $\#\left(\mathbb{P}^{n-1}, \Delta\right)(\mathbb{Q})_{\leqslant B}^{+}$.

Finally, we will explain why this result suffices to prove Theorem 1.1. 


\section{Calculating \# $M_{a, t}(B)$}

Let us first fix the framework of the circle method.

Let $T$ be $\mathbb{R} / \mathbb{Z}$. For $0<\Delta \leqslant 1$ and $P \geqslant 1$ (we always suppose $B \geqslant 1$ ), we define $\mathfrak{M}(\Delta, q, a)$ as the image in $T$ of $\left\{\alpha \in \mathbb{R}:|\alpha-a / q|<P^{\Delta-2}\right\}$ with $a, q \in \mathbb{Z}$ and

$$
\mathfrak{M}(\Delta)=\bigcup_{\substack{1 \leqslant a \leqslant q \leqslant P^{\Delta} \\ \operatorname{gcd}(a, q)=1}} \mathfrak{M}(\Delta, q, a) .
$$

We call $\mathfrak{M}(\Delta)$ the union of the major arcs and $T \backslash \mathfrak{M}(\Delta)=\mathfrak{m}(\Delta)$ the union of the minor arcs. We shall clarify the constraint on the constant $\Delta$ and the dependence of $P$ on $B$ in Proposition 3.7 and Theorem 3.8.

The circle method calculates $\# M_{\underline{a}, t}(B)$ by integrating an exponential sum over $T$, namely

$$
\# M_{\underline{a}, t}(B)=\int_{T} \sum_{\substack{1 \leqslant\left|a_{i} x_{i}^{2} y_{i}^{3}\right| \leqslant B \\ i=0, \ldots, n}}\left(\prod_{i=0}^{n} \mu_{i}^{\prime}\left(y_{i}\right)\right) e(\alpha f(\underline{x}, \underline{y})) d \alpha,
$$
where $f(\underline{x}, \underline{y})=\sum_{i=0}^{n} a_{i} x_{i}^{2} y_{i}^{3}-t$. We will denote the integrand of (2) by $E(\alpha)$ and
will set

$$
S_{i}(\alpha)=\sum_{1 \leqslant\left|a_{i} x^{2} y^{3}\right| \leqslant B} \mu_{i}^{\prime}(y) e\left(\alpha a_{i} x^{2} y^{3}\right) .
$$

Therefore,

$$
E(\alpha)=e(-\alpha t) \prod_{i=0}^{n} S_{i}(\alpha) .
$$

As usual, the integral over $\mathfrak{M}(\Delta)$ will provide the main term while the integral over $\mathfrak{m}(\Delta)$ will only contribute to the error term.

Major arcs. We refer to [Schmidt 1984, Section 5; Davenport 2005, Chapter 4] for avoid conflict with theorems. (Many authors improperly cite a detailed description of the circle method over the major arcs for the classical case of diagonal equations. In order to apply this to $\int_{\mathfrak{M}(\Delta)} E(\alpha) d \alpha$, we will first fix $\underline{y}$ and thus consider the diagonal equation $f(\underline{x}, \underline{y})=f_{y}(\underline{x})=0$; afterwards we will take the sum of the obtained expression over all admitted $y$.

Since we fix $\underline{y}$, we only look at $x_{i}$ satisfying $1 /\left|a_{i} y_{i}^{3}\right|^{1 / 2} \leqslant\left|x_{i}\right| \leqslant\left(B /\left|a_{i} y_{i}^{3}\right|\right)^{1 / 2}$. Most of the time, it suffices to consider only positive $x_{i}$; we will denote the corresponding interval for positive $x_{i}$ with $D_{i}$, that is,

$$
D_{i}=\left[1 /\left|a_{i} y_{i}^{3}\right|^{1 / 2}, B^{1 / 2} /\left|a_{i} y_{i}^{3}\right|^{1 / 2}\right] .
$$


We will also use the notation

$$
B_{a_{i}, y_{i}}=B^{1 / 2} /\left|a_{i} y_{i}^{3}\right|^{1 / 2} .
$$

Note that since we consider only $\underline{y}$ with $1 \leqslant\left|y_{i}^{3}\right| \leqslant B$, we have $1 \leqslant B_{a_{i}, y_{i}} \leqslant B^{1 / 2}$ for each $i \in\{0, \ldots, n\}$.

Because we first wish to examine the exponential sum $E(\alpha)$ (for $\alpha \in \mathfrak{M}(\Delta)$ ) for some $\underline{y}$ fixed, we denote this part of $E(\alpha)$ by

$$
E_{\underline{y}}(\alpha)=\sum_{\substack{1 /\left|a_{i} y_{i}^{3}\right|^{1 / 2} \leqslant\left|x_{i}\right| \leqslant B_{a_{i}, y_{i}} \\ i=0, \ldots, n}} e\left(\alpha f_{\underline{y}}(\underline{x})\right) .
$$

Furthermore, for every positive integer $q$ and every integer $a$ relatively prime to $q$, we define

$$
\sigma_{\underline{y}}\left(\frac{a}{q}\right)=q^{-(n+1)} \sum_{\underline{z} \in(\mathbb{Z} / q \mathbb{Z})^{n+1}} e\left(\frac{a f_{\underline{y}}(\underline{z})}{q}\right),
$$

and for every $\beta \in \mathbb{R}$,

$$
\tau_{\underline{y}, B}(\beta)=\int_{D_{0}} \cdots \int_{D_{n}} e\left(\beta f_{\underline{y}}(\underline{x})\right) d \underline{x} .
$$

Proposition 3.1. For $\alpha=a / q+\beta \in \mathfrak{M}(\Delta ; q, a)$, we have

$$
E_{\underline{y}}(\alpha)=2^{n+1} \sigma_{\underline{y}}\left(\frac{a}{q}\right) \tau_{\underline{y}, B}(\beta)+O\left(q \frac{\sum_{i=0}^{n}\left|a_{i} y_{i}^{3}\right|^{1 / 2}}{\prod_{i=0}^{n}\left|a_{i} y_{i}^{3}\right|^{1 / 2}} B^{(n+2) / 2} P^{\Delta-2}\right)
$$

under the condition $B P^{\Delta-2} \geqslant 1$ on $P$ and $\Delta$.

Proof. Combining positive and negative signs of $x_{i}$, we have

$$
E_{\underline{y}}(\alpha)=2^{n+1} e(-\alpha t) \prod_{i=0}^{n} \sum_{x_{i} \in D_{i}} e\left(\alpha a_{i} x_{i}^{2} y_{i}^{3}\right) .
$$

For $\alpha=a / q+\beta$, the inner sum over $x_{i}$ equals

$$
\sum_{1 \leqslant z_{i} \leqslant q} e\left(\frac{a a_{i} z_{i}^{2} y_{i}^{3}}{q}\right) \sum_{\substack{v_{i} \in \mathbb{Z} \\ q v_{i}+z_{i} \in D_{i}}} e\left(\beta a_{i}\left(q v_{i}+z_{i}\right)^{2} y_{i}^{3}\right) .
$$

Euler's summation formula (in its simplest version) implies

$$
\sum_{X \leqslant q v+z \leqslant Y} e\left(\zeta(q v+z)^{2}\right)=\frac{1}{q} \int_{X}^{Y} e\left(\zeta \eta^{2}\right) d \eta+O\left(1+\frac{Y}{q}|\zeta| q Y\right)
$$

for any real numbers $0 \leqslant X<Y, \zeta \in \mathbb{R}, q, z \in \mathbb{N}$. Taking $Y=B_{a_{i}, y_{i}}, \zeta=\beta a_{i} y_{i}^{3}$ 
and recalling the definition of $D_{i}$ in (3), we can rewrite (8) as

$$
\sum_{1 \leqslant z_{i} \leqslant q} e\left(\frac{a a_{i} z_{i}^{2} y_{i}^{3}}{q}\right)\left(\frac{1}{q} \int_{D_{i}} e\left(\beta a_{i} x_{i}^{2} y_{i}^{3}\right) d x_{i}+O(1+|\beta| B)\right) .
$$

We substitute these expressions successively back into (7) and obtain the desired main term. Using the trivial upper bounds

$$
\left|\sum_{x_{i} \in D_{i}} e\left(\alpha a_{i} x_{i}^{2} y_{i}^{3}\right)\right|+\left|\frac{1}{q} \sum_{1 \leqslant z_{i} \leqslant q} e\left(\frac{a a_{i} z_{i}^{2} y_{i}^{3}}{q}\right) \int_{D_{i}} e\left(\beta a_{i} x_{i}^{2} y_{i}^{3}\right) d x_{i}\right| \ll B_{a_{i}, y_{i}},
$$

we get the total error term $O\left(q(1+|\beta| B) \max _{0 \leqslant i \leqslant n} \prod_{j \neq i} B_{a_{j}, y_{j}}\right)$. Using (4) and $1+|\beta| B \ll P^{\Delta-2} B$, we complete the proof.

From this result, we can now derive an expression for the integral of $E_{y}(\alpha)$ over $\mathfrak{M}(\Delta)$ by first integrating the expression for $E_{y}(\alpha)$ obtained in Proposition 3.1 over $\mathfrak{M}(\Delta ; q, a)$ and then summing over all admitted $a$ and $q$.

We first define

$$
\mathfrak{I}_{\underline{\varepsilon}, t, B}(L)=\int_{|\gamma|<L} e(-\gamma t / B) d \gamma \int_{\left[B^{-1 / 2}, 1\right]^{n+1}} e\left(\gamma \sum_{i=0}^{n} \varepsilon_{i} x_{i}^{\prime 2}\right) d \underline{x}^{\prime},
$$

(where $\left.\varepsilon_{i}=\operatorname{sgn}\left(a_{i} y_{i}\right)\right)$ and

$$
\mathfrak{S}_{\underline{y}, \underline{a}, t}(L)=\sum_{q \leqslant L} \sum_{\substack{0<\frac{a}{\xi} \leqslant 1 \\ \operatorname{gcd}(a, q)=1}} \sigma_{\underline{y}}\left(\frac{a}{q}\right) .
$$

We have

$$
\int_{|\beta|<P^{\Delta-2}} \tau_{\underline{y}, B}(\beta) d \beta=\frac{B^{(n-1) / 2}}{\prod_{i=0}^{n}\left|a_{i} y_{i}^{3}\right|^{1 / 2}} \mathfrak{I}_{\underline{\varepsilon}, t, B}\left(B P^{\Delta-2}\right),
$$

and therefore

$$
\begin{aligned}
& \int_{\mathfrak{M}(\Delta)} E_{\underline{y}}(\alpha) d \alpha=\frac{2^{n+1} \mathfrak{S}_{\underline{y}, \underline{a}, t}\left(P^{\Delta}\right) \mathfrak{I}_{\underline{\varepsilon}, t, B}\left(B P^{\Delta-2}\right)}{\prod_{i=0}^{n}\left|a_{i} y_{i}^{3}\right|^{1 / 2}} \cdot B^{(n-1) / 2} \\
&+O\left(\frac{\sum_{i=0}^{n}\left|a_{i} y_{i}^{3}\right|^{1 / 2}}{\prod_{i=0}^{n}\left|a_{i} y_{i}^{3}\right|^{1 / 2}} B^{(n+2) / 2} P^{5 \Delta-4}\right) .
\end{aligned}
$$

Note that the integral $\mathfrak{I}_{\underline{\varepsilon}, t, B}(L)$ only depends on the signs of $\underline{y}$ and $\underline{a}$ and no longer on their actual values.

Next, we make the coefficient of $B^{(n-1) / 2}$ in this expression independent of $B$. We first focus on the factor $\mathfrak{S}_{\underline{y}, \underline{a}, t}\left(P^{\Delta}\right)$. 
The singular series.

Lemma 3.2. We have

$$
\left|\sigma_{\underline{y}}\left(\frac{a}{q}\right)\right| \ll q^{-(n+1) / 2} \cdot \prod_{i=0}^{n} \operatorname{gcd}\left(a_{i} y_{i}^{3}, q\right)^{1 / 2} .
$$

Proof. Using elementary properties of generalized Gauss sums (see for example [Berndt et al. 1998, Chapter 1]), we obtain for positive integers $a$ and $c$ that

$$
\left|\sum_{n=0}^{c-1} e\left(\frac{a n^{2}}{c}\right)\right| \ll \operatorname{gcd}(a, c)^{1 / 2} \sqrt{c} .
$$

Applying this to (5) implies the statement.

Corollary 3.3. For $n \geqslant 4$, the series

$$
\mathfrak{S}_{\underline{y}, \underline{a}, t}=\sum_{q=1}^{\infty} \sum_{\substack{0<a / q \leqslant 1 \\ \operatorname{gcd}(a, q)=1}} \sigma_{\underline{y}}\left(\frac{a}{q}\right),
$$

called the singular series, converges absolutely. In particular, we have

$$
\mathfrak{S}_{\underline{y}, \underline{a}, t} \ll \frac{\prod_{i=0}^{n}\left|a_{i} y_{i}^{3}\right|^{1 / 2+\varepsilon}}{\operatorname{lcm}\left(a_{0} y_{0}^{3}, \ldots, a_{n} y_{n}^{3}\right)^{1 / 2}}
$$

and

$$
\mathfrak{S}_{\underline{y}, \underline{a}, t}\left(P^{\Delta}\right)=\mathfrak{S}_{\underline{y}, \underline{a}, t}+O\left(\frac{\prod_{i=0}^{n}\left|a_{i} y_{i}^{3}\right|^{1 / 2+\varepsilon}}{\operatorname{lcm}\left(a_{0} y_{0}^{3}, \ldots, a_{n} y_{n}^{3}\right)^{1 / 2}} \cdot P^{\Delta(-n+3) / 2}\right)
$$

for any $\varepsilon>0$.

Proof. From the previous lemma, we deduce that

$$
\begin{aligned}
\mathfrak{S}_{\underline{y}, \underline{a}, t} & \ll \sum_{q=1}^{\infty} q^{-(n-1) / 2} \prod_{i=0}^{n} \operatorname{gcd}\left(a_{i} y_{i}^{3}, q\right)^{1 / 2} \\
& \ll \sum_{\substack{d_{i} \mid a_{i} y_{i}^{3} \\
i=0, \ldots, n}}\left(d_{0} \cdots d_{n}\right)^{1 / 2} \sum_{\substack{q=1 \\
\operatorname{lcm}\left(d_{0}, \ldots, d_{n}\right) \mid q}}^{\infty} q^{-(n-1) / 2} \\
& \ll \sum_{\substack{d_{i} \mid a_{i} y_{i}^{3} \\
i=0, \ldots, n}} \frac{\left(d_{0} \cdots d_{n}\right)^{1 / 2}}{\operatorname{lcm}\left(d_{0}, \ldots, d_{n}\right)^{(n-1) / 2}} \sum_{q=1}^{\infty} q^{-(n-1) / 2} .
\end{aligned}
$$


Since $n \geqslant 4$, the latter expression converges and we get

$$
\begin{aligned}
\mathfrak{S}_{\underline{y}, \underline{a}, t} & \ll \sum_{\substack{d_{i} \mid a_{i} y_{i}^{3} \\
i=0, \ldots, n}} \frac{\left(d_{0} \cdots d_{n}\right)^{1 / 2}}{\operatorname{lcm}\left(d_{0}, \ldots, d_{n}\right)^{(n-1) / 2}} \\
& \ll \frac{\prod_{i=0}^{n}\left|a_{i} y_{i}^{3}\right|^{1 / 2+\varepsilon}}{\operatorname{lcm}\left(a_{0} y_{0}^{3}, \ldots, a_{0} y_{0}^{3}\right)^{1 / 2}}
\end{aligned}
$$

for any $\varepsilon>0$. Moreover, we obtain in the same way that

$$
\begin{aligned}
\left|\mathfrak{S}_{\underline{y}, \underline{a}, t}-\mathfrak{S}_{\underline{y}, \underline{a}, t}\left(P^{\Delta}\right)\right| & \leqslant \sum_{q>P^{\Delta}} q^{-(n-1) / 2} \prod_{i=0}^{n} \operatorname{gcd}\left(a_{i} y_{i}^{3}, q\right)^{1 / 2} \\
& \ll \sum_{\substack{d_{i} \mid a_{i} y_{i}^{3} \\
0 \leqslant i \leqslant n}} \frac{\left(d_{0} \cdots d_{n}\right)^{1 / 2}}{\operatorname{lcm}\left(d_{0}, \ldots, d_{n}\right)^{(n-1) / 2}} \sum_{\substack{q>P^{\Delta} \\
\operatorname{lcm}\left(d_{0}, \ldots, d_{n}\right) \mid q}}^{\infty} q^{-(n-1) / 2} \\
& \ll \frac{\prod_{i=0}^{n}\left|a_{i} y_{i}^{3}\right|^{1 / 2+\varepsilon}}{\operatorname{lcm}\left(a_{0} y_{0}^{3}, \ldots, a_{n} y_{n}^{3}\right)^{1 / 2}} \cdot P^{\Delta(-n+3) / 2} .
\end{aligned}
$$

Remark 3.4. One can prove (see for example [Davenport 2005, Lemmas 5.2-5.3]) for $n \geqslant 4$ that $\mathfrak{S}_{\underline{y}, \underline{a}, t}$ can be written as an Euler product of $p$-adic densities

$$
\lim _{l \rightarrow \infty} \frac{\#\left\{\left(x_{0}, \ldots, x_{n}\right) \in\left(\mathbb{Z} / p^{l} \mathbb{Z}\right)^{n+1}: \sum_{i=0}^{n} a_{i} y_{i}^{3} x_{i}^{2} \equiv t \bmod p^{l}\right\}}{p^{l n}} .
$$

The singular integral. Examining $\mathfrak{I}_{\underline{\varepsilon}, t, B}\left(B P^{\Delta-2}\right)$ in (9), we have the following proposition.

Proposition 3.5. For $n \geqslant 3$, we have

$$
\mathfrak{I}_{\underline{\varepsilon}, t, B}\left(B P^{\Delta-2}\right)=\mathfrak{I}_{\underline{\varepsilon}, t, B}+O\left(B^{(1-n) / 2} P^{(\Delta-2)(1-n) / 2}\right)
$$

with

$$
\mathfrak{I}_{\underline{\varepsilon}, t, B}=\int_{-\infty}^{+\infty} e(-\gamma t / B) d \gamma \int_{\left[B^{-1 / 2}, 1\right]^{n+1}} e\left(\gamma \sum_{i=0}^{n} \varepsilon_{i} x_{i}^{2}\right) d \underline{x}
$$

under the condition $B P^{\Delta-2} \geqslant 1$.

Proof. As proved in [Davenport 2005, Proof of Theorem 4.1], we have

$$
\left|\int_{B^{-1 / 2}}^{1} e\left(\gamma \varepsilon_{i} x_{i}^{2}\right) d x_{i}\right| \ll \min \left\{1,|\gamma|^{-1 / 2}\right\}
$$

and thus

$$
\left|\int_{\left[B^{-1 / 2}, 1\right]^{n+1}} e\left(\gamma \sum_{i=0}^{n} \varepsilon_{i} x_{i}^{2}\right) d \underline{x}\right| \ll \min \left\{1,|\gamma|^{-1 / 2}\right\}^{n+1} .
$$


This implies that the integral $\mathfrak{I}_{\underline{\varepsilon}, t, B}$ converges, since

$$
\left|\mathfrak{I}_{\underline{\varepsilon}, t, B}\right| \ll \int_{-\infty}^{+\infty} \min \left\{1,|\gamma|^{-1 / 2}\right\}^{n+1} d \gamma<+\infty .
$$

Also,

$$
\begin{aligned}
\left|\mathfrak{I}_{\underline{\varepsilon}, t, B}\left(B P^{\Delta-2}\right)-\mathfrak{I}_{\underline{\varepsilon}, t, B}\right| & \ll \int_{|\gamma|>B P^{\Delta-2}}|\gamma|^{-(n+1) / 2} d \gamma \\
& \ll B^{(1-n) / 2} P^{(\Delta-2)(1-n) / 2} .
\end{aligned}
$$

Defining the singular integral as

$$
\mathfrak{I}_{\underline{\varepsilon}}=\int_{-\infty}^{+\infty} d \gamma \int_{[0,1]^{n+1}} e\left(\gamma \sum_{i=0}^{n} \varepsilon_{i} x_{i}^{2}\right) d \underline{x},
$$

it follows from the last proof that this integral is also convergent.

Lemma 3.6. It holds that $\mathfrak{I}_{\underline{\varepsilon}, t, B} \rightarrow \mathfrak{I}_{\underline{\varepsilon}}$ as $B$ goes to infinity.

Proof. We have

$$
\begin{aligned}
\left|\mathfrak{I}_{\underline{\varepsilon}, t, B}-\mathfrak{I}_{\underline{\varepsilon}}\right| \leqslant \int_{-\infty}^{+\infty}|(e(-\gamma t / B)-1)| d \gamma\left|\int_{\left[B^{-1 / 2}, 1\right]^{n+1}} e\left(\gamma \sum_{i=0}^{n} \varepsilon_{i} x_{i}^{2}\right) d \underline{x}\right| \\
\quad+\int_{-\infty}^{+\infty} d \gamma\left|\int_{\left(\left[B^{-1 / 2}, 1\right]^{n+1}\right)^{c}} e\left(\gamma \sum_{i=0}^{n} \varepsilon_{i} x_{i}^{2}\right) d \underline{x}\right| \\
=I_{1}(B, t)+I_{2}(B),
\end{aligned}
$$

where $\left(\left[B^{-1 / 2}, 1\right]^{n+1}\right)^{c}$ denotes the complement of $\left[B^{-1 / 2}, 1\right]^{n+1}$ in the hypercube $[0,1]^{n+1}$.

Since $|(e(-\gamma t / B)-1)|=2\left|\sin \left(\pi \gamma t B^{-1}\right)\right| \leqslant \min \left\{2,2 \pi|\gamma||t| B^{-1}\right\}$, we obtain the following for $I_{1}(B, t)$, recalling (14):

$$
I_{1}(B, t) \ll \int_{-\infty}^{+\infty} \min \left\{1, \pi|\gamma||t| B^{-1}\right\} \cdot \min \left\{1,|\gamma|^{-1 / 2}\right\}^{n+1} d \gamma
$$

Splitting up the latter integral into three parts according to the appropriate range of $\gamma$, we get $I_{1}(B, t) \ll|t| B^{-1}$ for $B$ big enough.

For $I_{2}(B)$, one has

$$
\left|\int_{0}^{1} e\left(\gamma \varepsilon_{i} x_{i}^{2}\right) d x_{i}\right| \ll \min \left\{1,|\gamma|^{-1 / 2}\right\}
$$

and

$$
\left|\int_{0}^{B^{-1 / 2}} e\left(\gamma \varepsilon_{i} x_{i}^{2}\right) d x_{i}\right| \ll \min \left\{B^{-1 / 2},|\gamma|^{-1 / 2}\right\} .
$$


Applying the exclusion-inclusion principle to $I_{2}(B)$ and observing the symmetric form of the integrand, we get

$$
I_{2}(B) \ll \sum_{i=1}^{n+1} \int_{-\infty}^{+\infty} \min \left\{B^{-1 / 2},|\gamma|^{-1 / 2}\right\}^{i} \cdot \min \left\{1,|\gamma|^{-1 / 2}\right\}^{n+1-i} d \gamma .
$$

It follows that $I_{2}(B) \ll B^{-1 / 2}$. Hence,

$$
\left|\mathfrak{I}_{\underline{\varepsilon}, t, B}-\mathfrak{I}_{\underline{\varepsilon}}\right| \ll_{t} B^{-1 / 2}
$$

for $B$ big enough, completing the proof.

Note that from Proposition 3.5 and (16), one has

$$
\mathfrak{I}_{\underline{\varepsilon}, t, B}\left(B P^{\Delta-2}\right)=\mathfrak{I}_{\underline{\varepsilon}}+O\left(B^{-1 / 2}+B^{(1-n) / 2} P^{(\Delta-2)(1-n) / 2}\right) .
$$

We now return to the integral of $E_{y}(\alpha)$ over the major arcs.

Proposition 3.7. For $n \geqslant 4$ and for any $\Delta$ with $0<\Delta<1 / 5$, there exists a $\delta>0$ so that

$$
\int_{\mathfrak{M}(\Delta)} E_{\underline{y}}(\alpha) d \alpha=\frac{2^{n+1} \mathfrak{S}_{\underline{y}, \underline{a}, t} \mathfrak{I}_{\underline{\underline{\varepsilon}}}}{\prod_{i=0}^{n}\left|a_{i} y_{i}^{3}\right|^{1 / 2}} \cdot B^{(n-1) / 2}+O_{\underline{y}, \underline{a}}\left(B^{(n-1) / 2-\delta}\right) .
$$

Proof. Substituting (12) and (17) into formula (9), we get

$$
\begin{aligned}
\int_{\mathfrak{M}(\Delta)} E_{\underline{y}}(\alpha) d \alpha=\frac{2^{n+1} \mathfrak{S}_{\underline{y}, \underline{a}, t} \mathfrak{I}_{\underline{\varepsilon}}}{\prod_{i=0}^{n}\left|a_{i} y_{i}^{3}\right|^{1 / 2}} \cdot B^{(n-1) / 2} \\
+O\left(\frac{\prod_{i=0}^{n}\left|a_{i} y_{i}^{3}\right|^{\varepsilon}}{\operatorname{lcm}\left(a_{0} y_{0}^{3}, \ldots, a_{n} y_{n}^{3}\right)^{1 / 2}} \cdot B^{(n-1) / 2} P^{\Delta(-n+3) / 2}\right. \\
\left.\quad+\frac{B^{(n-2) / 2}+P^{(\Delta-2)(1-n) / 2}}{\prod_{i=0}^{n}\left|a_{i} y_{i}^{3}\right|^{1 / 2}}+\frac{\sum_{i=0}^{n}\left|a_{i} y_{i}^{3}\right|^{1 / 2}}{\prod_{i=0}^{n}\left|a_{i} y_{i}^{3}\right|^{1 / 2}} \cdot B^{(n+2) / 2} P^{5 \Delta-4}\right) .
\end{aligned}
$$

For this expression to be nontrivial, we have to determine $P=P(B)$ and $\Delta$ properly (under the condition $B P^{\Delta-2} \geqslant 1$ ) so that the error term is $O_{y, \underline{a}}\left(B^{(n-1) / 2-\delta}\right.$ ) for some $\delta>0$. Taking $P=B^{1 / 2}$ and $0<\Delta<1 / 5$ is satisfactory.

We can now prove our estimate for the major arcs.

Theorem 3.8. For $n \geqslant 4$ and for any $\Delta$ with $0<\Delta<1 / 15$, there exists a $\delta>0$ so that

$$
\int_{\mathfrak{M}(\Delta)} E(\alpha) d \alpha=C_{\underline{a}, t} \cdot B^{(n-1) / 2}+O\left(B^{(n-1) / 2-\delta}\right),
$$

where

$$
C_{\underline{a}, t}=2^{n+1} \sum_{\underline{y} \in \mathbb{Z}_{0}^{n+1}}\left(\prod_{i=0}^{n} \mu_{i}^{\prime}\left(y_{i}\right)\right) \frac{\mathfrak{S}_{\underline{y}, \underline{a}, t} \mathfrak{I}_{\underline{\varepsilon}}}{\prod_{i=0}^{n}\left|a_{i} y_{i}^{3}\right|^{1 / 2}}
$$


with $\mathfrak{S}_{\underline{y}, \underline{a}, t}$ and $\mathfrak{I}_{\underline{\varepsilon}}$ as defined above.

Proof. We sum (19) over all admitted $y_{i}$ such that $1 \leqslant\left|y_{i}^{3}\right| \leqslant B, i \in\{0, \ldots, n\}$, and denote the sum of the coefficients of the main term by $C_{\underline{a}, t}(B)$.

We obtain, using (11),

$$
\frac{\mathfrak{S}_{\underline{y}, \underline{a}, t}}{\prod_{i=0}^{n}\left|a_{i} y_{i}^{3}\right|^{1 / 2}} \ll \frac{\prod_{i=0}^{n}\left|a_{i} y_{i}^{3}\right|^{\varepsilon}}{\operatorname{lcm}\left(a_{0} y_{0}^{3}, \ldots, a_{n} y_{n}^{3}\right)^{1 / 2}}
$$

for any $\varepsilon>0$. We have

$$
\begin{aligned}
\sum_{\max _{0 \leqslant i \leqslant n}\left|y_{i}^{3}\right| \geqslant B} \frac{\prod_{i=0}^{n}\left|a_{i} y_{i}^{3}\right|^{\varepsilon}}{\operatorname{lcm}\left(a_{0} y_{0}^{3}, \ldots, a_{n} y_{n}^{3}\right)^{1 / 2}} & \ll \sum_{\max _{0 \leqslant i \leqslant n}\left|y_{i}^{3}\right| \geqslant B} \frac{1}{\operatorname{lcm}\left(a_{0} y_{0}^{3}, \ldots, a_{n} y_{n}^{3}\right)^{1 / 2-(n+1) \varepsilon}} \\
& \ll \sum_{\max _{0 \leqslant i \leqslant n}\left|y_{i}^{3}\right| \geqslant B} \frac{1}{\operatorname{lcm}\left(y_{0}, \ldots, y_{n}\right)^{3 / 2-3(n+1) \varepsilon}} \\
& \ll \sum_{N^{3} \geqslant B} \frac{\#\left\{\left(y_{0}, \ldots, y_{n}\right): \operatorname{lcm}\left(y_{0}, \ldots, y_{n}\right)=N\right\}}{N^{3 / 2-3(n+1) \varepsilon}} \\
& \ll B^{-1 / 6+(n+1) \varepsilon}
\end{aligned}
$$

for any $\varepsilon>0$. This allows us to replace $C_{\underline{a}, t}(B)$ by $C_{\underline{a}, t}$.

We now turn to the error term in (19), summing over all admitted values of $\underline{y}$ and putting $P=B^{1 / 2}$ as before.

The first error term can be treated as the main term. The coefficients of the third and fourth error terms will also converge without any extra conditions. Moreover, the upper bound can be made independent of the $a_{i}$. For the last error term however, the coefficient will asymptotically contribute $O\left(B^{1 / 3}\right)$.

This means the extra condition

$$
\frac{1}{3}+\frac{n+2}{2}+\frac{5 \Delta-4}{2}<\frac{n-1}{2} \Leftrightarrow \Delta<\frac{1}{15}
$$

has to be satisfied for the error term to behave properly.

Note that (20) and (21) also provides a uniform upper bound of $C_{\underline{a}, t}$, that is, $C_{\underline{a}, t} \leqslant C$, independently of $\underline{a}$ and $t$.

Minor arcs. The goal of this section is to prove the following theorem.

Theorem 3.9. For $n \geqslant 4$, there exists a $\delta>0$ so that

$$
\int_{\mathfrak{m}(\Delta)} E(\alpha) d \alpha=O\left(B^{(n-1) / 2-\delta}\right) .
$$


To treat the integral over the minor arcs, we will not fix $y$ but examine the whole equation at once. Recall that

$$
E(\alpha)=e(-\alpha t) \prod_{i=0}^{n} S_{i}(\alpha)=e(-\alpha t) \prod_{i=0}^{n} \sum_{1 \leqslant\left|a_{i} x^{2} y^{3}\right| \leqslant B} \mu_{i}^{\prime}(y) e\left(\alpha a_{i} x^{2} y^{3}\right) .
$$

Using Hölder's inequality repeatedly, we get for $n \geqslant 4$,

$$
\left|\int_{\mathfrak{m}(\Delta)} E(\alpha) d \alpha\right| \leqslant \sup _{\alpha \in \mathfrak{m}(\Delta)}\left(\left|S_{0}(\alpha)\right| \cdots\left|S_{n-4}(\alpha)\right|\right) \max _{j=n-3, \ldots, n} \int_{0}^{1}\left|S_{j}(\alpha)\right|^{4} d \alpha .
$$

To obtain a good upper bound of this expression, we first examine $\int_{0}^{1}\left|S_{j}(\alpha)\right|^{4} d \alpha$.

Lemma 3.10. For any $\varepsilon>0$, we have

$$
\int_{0}^{1}\left|S_{j}(\alpha)\right|^{4} d \alpha \ll_{\varepsilon} B^{1+\varepsilon} .
$$

Proof. From now on, we will concentrate on the part of the sum where the variables are positive. This will suffice to prove the theorem because of the symmetry.

Let

$$
S_{Y}(\alpha)=\sum_{Y<y \leqslant 2 Y} \mu_{j}^{\prime}(y) \sum_{1 \leqslant x \leqslant B_{a_{j}, y}} e\left(\alpha a_{j} x^{2} y^{3}\right)
$$

be the contribution to $S_{j}(\alpha)$ for $Y<y \leqslant 2 Y$. Using Cauchy's inequality, it follows that

$$
\begin{aligned}
\int_{0}^{1}\left|S_{Y}(\alpha)\right|^{4} d \alpha & \ll Y \int_{0}^{1}\left|S_{Y}(\alpha)\right|^{2} \sum_{Y<y \leqslant 2 Y} \mu_{j}^{\prime}(y)\left|\sum_{1 \leqslant x \leqslant B_{a_{j}, y}} e\left(\alpha a_{j} x^{2} y^{3}\right)\right|^{2} d \alpha \\
& \ll Y \sum_{Y<y_{1}, y_{2}, y_{3} \leqslant 2 Y} \sum_{\substack{1 \leqslant x_{1} \leqslant B_{a_{j}, y_{1}} \\
1 \leqslant x_{2} \leqslant B_{a_{j}, y_{2}} \\
1 \leqslant x_{3}, x_{4} \leqslant B_{a_{j}, y_{3}}}} \int_{0}^{1} e\left(\alpha a_{j} G(\underline{x}, \underline{y})\right) d \alpha \\
& \leqslant Y \cdot \# Z(Y, B),
\end{aligned}
$$

with $G(\underline{x}, \underline{y})=y_{3}^{3}\left(x_{4}^{2}-x_{3}^{2}\right)+x_{1}^{2} y_{1}^{3}-x_{2}^{2} y_{2}^{3}$ and $Z(Y, B)=\left\{(\underline{x}, \underline{y}) \in \mathbb{Z}_{0}^{7}: y_{3}^{3}\left(x_{3}^{2}-x_{4}^{2}\right)=\right.$ $\left.x_{1}^{2} y_{1}^{3}-x_{2}^{2} y_{2}^{3}, 1 \leqslant x_{i}<B_{Y}, Y<y_{j} \leqslant 2 Y\right\}$, where $B_{Y}=\left(B / Y^{3}\right)^{1 / 2}$.

If we make a distinction between solutions $(\underline{x}, y) \in \mathbb{Z}_{0}^{7}$ of $G(\underline{x}, y)=0$ for which $x_{1}^{2} y_{1}^{3}-x_{2}^{2} y_{2}^{3}=0$ or not, it follows that both sets contain $O\left(Y^{-1} \cdot B^{1+\varepsilon}\right)$ solutions. Hence, we conclude that $\# Z(Y, B) \ll_{\varepsilon} Y^{-1} \cdot B^{1+\varepsilon}$, and thus

$$
\int_{0}^{1}\left|S_{Y}(\alpha)\right|^{4} d \alpha \ll_{\varepsilon} B^{1+\varepsilon} .
$$


Summing over all intervals $(Y, 2 Y]$ with $Y=2^{k} \ll B^{1 / 3}$ and applying Cauchy's inequality twice on $\left|S_{j}(\alpha)\right|^{4}=\left|\sum_{Y=2^{k} \ll B^{1 / 3}} S_{Y}(\alpha)\right|^{4}$, we get

$$
\int_{0}^{1}\left|S_{j}(\alpha)\right|^{4} d \alpha \ll B^{3 \varepsilon^{\prime}} \sum_{Y=2^{k} \ll B^{1 / 3}} \int_{0}^{1}\left|S_{Y}(\alpha)\right|^{4} d \alpha \ll B^{3 \varepsilon^{\prime}} \sum_{Y=2^{k} \ll B^{1 / 3}} B^{1+\varepsilon}=B^{1+\varepsilon^{\prime \prime}}
$$

which completes the proof.

Remark 3.11. Recalling the expression for $\# M_{\underline{a}, t}(B)$ in (2) and putting $n=3$, $\underline{a}=(1,1,1,1), t=0$ and $\mu_{i}^{\prime}(\cdot)=\mu^{2}(\cdot)$ for each $i$, this lemma implies that the equation $n_{1}+n_{2}=n_{3}+n_{4}$, where $n_{i}$ is squareful and $1 \leqslant\left|n_{i}\right| \leqslant B$ for each $i \in\{1,2,3,4\}$, has $O\left(B^{1+\varepsilon}\right)$ solutions.

In order to handle the first part of (22), namely $\sup _{\alpha \in \mathfrak{m}(\Delta)}\left(\left|S_{0}(\alpha)\right| \cdots\left|S_{n-4}(\alpha)\right|\right)$, we will prove the following proposition.

Proposition 3.12. Let $\alpha \in \mathfrak{m}(\Delta)$. Then there exists a $\delta>0$ such that

$$
\left|S_{i}(\alpha)\right| \ll B^{1 / 2-\delta} .
$$

Proof. Let $\psi>0$. We may henceforth assume that $\left|a_{i}\right| \leqslant B^{\psi}$, since otherwise the trivial upper bound yields

$$
\left|S_{i}(\alpha)\right| \leqslant \sum_{y=1}^{\infty} \sqrt{\frac{B}{a_{i} y^{3}}} \ll B^{(1-\psi) / 2},
$$

which is satisfactory. Similarly, we may assume that $y \leqslant B^{\psi}$ in $S_{i}(\alpha)$. Thus, we have

$$
\left|S_{i}(\alpha)\right| \ll B^{(1-\psi) / 2}+\sum_{y \leqslant B^{\psi}} \mu_{i}^{\prime}(y)\left|T_{\underline{y}}(\alpha)\right|,
$$

with, if we set $X=\sqrt{B /\left(\left|a_{i}\right| y^{3}\right)}$,

$$
T_{\underline{y}}(\alpha)=\sum_{x \leqslant X} e\left(\alpha a_{i} y^{3} x^{2}\right) .
$$

Since $\left|a_{i}\right| y^{3} x^{2} \leqslant B$, in particular $X \geqslant B^{1 / 2-2 \psi}$. Using the usual squaring and differencing approach (see for example [Davenport 2005, Chapter 3]), we obtain

$$
\begin{aligned}
\left|T_{\underline{y}}(\alpha)\right|^{2} & \leqslant \sum_{|h| \leqslant X}\left|\sum_{\substack{x \\
x, x+h \leqslant X}} e\left(2 \alpha a_{i} y^{3} h x\right)\right| \\
& \ll \sum_{|h| \leqslant X} \min \left\{X,\left\|2 \alpha a_{i} y^{3} h\right\|^{-1}\right\} \ll X+B^{\varepsilon} \cdot \sum_{y \leqslant Y} \min \left\{X,\|\alpha y\|^{-1}\right\},
\end{aligned}
$$

where $Y=2\left|a_{i}\right| y^{3} X$ and $\|a\|=\min \{|\beta| \in \mathbb{R}: \beta \equiv a \bmod 1\}$ for any real number $a$. In order to estimate the sum over $y$, we will use the following lemma. 
Lemma 3.13 (Separation lemma). Let $P, Q \geqslant 1$ be reals, $\alpha \in T$ and $a, q \in \mathbb{Z}$ with $\operatorname{gcd}(a, q)=1$ and $|\alpha-a / q|<q^{-2}$. Then

$$
\sum_{x \leqslant P} \min \left\{\frac{P Q}{x},\|\alpha x\|^{-1}\right\} \ll P Q\left(q^{-1}+Q^{-1}+q(P Q)^{-1}\right) \log (2 q P) .
$$

Proof. A full proof is given in [Vaughan 1997, Lemma 2.2].

Choosing $P=Y$ and $Q=X$, Lemma 3.13 implies

$$
\begin{aligned}
\left|T_{\underline{y}}(\alpha)\right|^{2} & \ll X+X Y B^{\varepsilon}\left(\frac{1}{q}+\frac{1}{X}+\frac{q}{X Y}\right) \\
& \ll X Y B^{2 \varepsilon}\left(\frac{1}{q}+\frac{1}{X}+\frac{q}{X Y}\right) \\
& \ll B^{1+2 \varepsilon}\left(\frac{1}{q}+B^{2 \psi-1 / 2}\right)+q B^{2 \varepsilon},
\end{aligned}
$$

since $X \leqslant Y$ and $X Y=2\left|a_{i}\right| y^{3} X^{2}=2 B$. Hence,

$$
\left|S_{i}(\alpha)\right| \ll B^{1 / 2-2 \psi}+B^{1 / 2+\varepsilon+\psi}\left(\frac{1}{\sqrt{q}}+B^{\psi-1 / 4}\right)+\sqrt{q} B^{\varepsilon+\psi} .
$$

According to Dirichlet, we can find $a, q \in \mathbb{Z}$ with $\operatorname{gcd}(a, q)=1$ and $q \leqslant B^{(2-\Delta) / 4}$ such that $|\alpha q-a|<1 / B^{(2-\Delta) / 4}=B^{(\Delta-2) / 4}$. (Note we also have $|\alpha-a / q|<1 / q^{2}$.) Furthermore, it is necessary that $q>B^{\Delta / 2}$ : otherwise, we would have $\alpha \in \mathfrak{M}(\Delta)$. With these boundaries for $q$ in (23), a suitable small choice for $\psi$ in terms of $\Delta$ leads to the statement.

We are now able to prove Theorem 3.9.

Proof of Theorem 3.9. Combining Proposition 3.12 and Lemma 3.10 in (22), we obtain

$$
\left|\int_{\mathfrak{m}(\Delta)} E(\alpha) d \alpha\right| \ll B^{(1 / 2-\delta)(n-3)} \cdot B^{1+\varepsilon} \leqslant B^{(n-1) / 2-\delta+\varepsilon}<B^{(n-1) / 2}
$$

for any $0<\varepsilon<\delta$.

\section{Towards the main problem}

Combining the previous results, we are able to prove the following theorem.

Theorem 4.1. For $n \geqslant 4$, there exists a $\delta>0$ so that

$$
\# M_{\underline{a}, t}(B)=C_{\underline{a}, t} \cdot B^{(n-1) / 2}+O\left(B^{(n-1) / 2-\delta}\right),
$$

with the constant $C_{\underline{a}, t}$ described in Theorem 3.8.

Proof. This follows directly from Theorem 3.8, Theorem 3.9 and (2). 
Remark 4.2. Note that the error term is independent of $\underline{a}$ and $t$ and recall we also proved $C_{\underline{a}, t}$ can be bounded uniformly independent of $\underline{a}$ and $t$. This implies that $\# M_{\underline{a}, t}(B) \leqslant C \cdot B^{(n-1) / 2}$ for some constant $C>0$. Indeed, when $B<1$, $M_{\underline{a}, t}(B)=\varnothing$, and for $B \geqslant 1$, it follows from Theorem 4.1 that

$$
\# M_{\underline{a}, t}(B) \leqslant C^{\prime} \cdot B^{(n-1) / 2}+C^{\prime \prime} \cdot B^{(n-1) / 2-\delta} \leqslant C \cdot B^{(n-1) / 2},
$$

where $C=2 \max \left\{C^{\prime}, C^{\prime \prime}\right\}$.

Going back to $M(B)$ (see definition on page 1021), we will now prove the following theorem.

Theorem 4.3. For $n \geqslant 4$, there exists an explicit constant $D$ and $a \delta>0$ such that

$$
\# M(B)=D \cdot B^{(n-1) / 2}+O\left(B^{(n-1) / 2-\delta}\right)
$$

as $B$ goes to infinity.

(The definition of the constant $D$ is given in Lemma 4.5; in the next section, we will give some indications about the interpretation of $D$.)

The only problem still left in proving Theorem 4.3 is to understand how we can tackle the additional gcd condition $\operatorname{gcd}\left(x_{0} y_{0}, \ldots, x_{n} y_{n}\right)=1$ on the solutions. Note that the Möbius inversion at hand leads to divisibility conditions on both $x_{i}$ and $y_{i}$ which have to be handled with care.

Let $\underline{e}=\left(e_{0}, \ldots, e_{n}\right) \in \mathbb{N}_{0}^{n+1}$ and $\underline{f}=\left(f_{0}, \ldots, f_{n}\right) \in \mathbb{N}_{0}^{n+1}$, where $f_{i}$ is squarefree for each $i \in\{0, \ldots, n\}$.

Definition. We denote the set

$\left\{(\underline{x}, \underline{y}) \in \mathbb{Z}_{0}^{2 n+2} \sum_{i=0}^{n} x_{i}^{2} y_{i}^{3}=0, \max _{0 \leqslant i \leqslant n}\left|x_{i}^{2} y_{i}^{3}\right| \leqslant B, e_{i}\left|x_{i}, f_{i}\right| y_{i}\right.$ and $\left.\prod_{i=0}^{n} \mu^{2}\left(\left|y_{i}\right|\right)=1\right\}$ by $N_{(\underline{e}, \underline{f})}(B)$.

Demanding that solutions in $N_{(1,1)}(B)$ satisfy $\operatorname{gcd}\left(x_{0} y_{0}, \ldots, x_{n} y_{n}\right)=1$ means we wish to leave out those solutions of $N_{(\underline{1}, \underline{1})}(B)$ for which there exists a prime $p$ and a subset $I \subset\{0, \ldots, n\}$ such that $p \mid x_{i}$ if $i \in I$ and $p \mid y_{i}$ if $i \notin I$ (or $i \in I^{c}$, where $I^{c}$ denotes the complement of $I$ in $\left.\{0, \ldots, n\}\right)$ in order to get to $M(B)$. Defining for a prime $p$ and subsets $I, J \subset\{0, \ldots, n\}$ the couple $\left(\underline{e}^{p, I}, \underline{f}^{p, J}\right)$ by $e_{i}^{p, I}=p$ for $i \in I$ and $e_{i}^{p, I}=1$ otherwise and analogously for $\underline{f}^{p, J}$, it hence follows that

$$
M(B)=N_{(\underline{1}, \underline{1})}(B) \backslash \bigcup_{(p, I)} N_{\left(\underline{e}^{p, I}, \underline{f}^{p, I^{c}}\right)}(B) .
$$

Notice that in this last union only a finite number of sets are nonempty since for a prime $p \geqslant \sqrt{B}$, we get $N_{\left(\underline{e}^{p, I}, \underline{f}^{p, I^{c}}\right)}(B)=\varnothing$. 
Definition. Let $S$ be a finite set of couples $(p, I)$. We can associate to $S$ a couple $(\underline{e}, f)$ as follows: defining for each prime $p$ the index sets $I_{p}=\cup_{(p, I) \in S} I$ and $J_{p}=$ $\cup_{(p, I) \in S} I^{c}$, the associated couple is given by $e_{i}=\prod_{\left\{p \mid i \in I_{p}\right\}} p$ and $f_{i}=\prod_{\left\{p \mid i \in J_{p}\right\}} p$. We then define $\mu(\underline{e}, \underline{f})=\sum_{n \geqslant 0}(-1)^{n} \#\{$ sets $S$ of cardinality $n$ with associated couple $(\underline{e}, \underline{f})\}$.

Observing (24) together with this definition, we have

$$
\# M(B)=\sum_{e=1}^{\infty} \sum_{\substack{(\underline{e}, \underline{f}) \in \mathbb{N}^{2 n+2} \\ e=\operatorname{gcd}\left(e_{i} f_{i}, i=0, \ldots, n\right)}} \mu(\underline{e}, \underline{f}) \cdot \# N_{(\underline{e}, \underline{f})}(B) .
$$

The following lemma collects some properties of $\mu$.

Lemma 4.4. There exists a function $\tilde{\mu}: \mathbb{Z}^{2 n+2} \rightarrow \mathbb{Z}$ such that

(i) $\mu(\underline{e}, \underline{f})=\prod_{p} \tilde{\mu}\left(v_{p}(\underline{e}), v_{p}(\underline{f})\right)$, where $v_{p}(\underline{e})=\left(v_{p}\left(e_{0}\right), \ldots, v_{p}\left(e_{n}\right)\right)($ and analogously for $\left.v_{p}(\underline{f})\right)$,

(ii) $\tilde{\mu}(\underline{m}, \underline{n})=0$ if $m_{i}=n_{i}=0$ and $(\underline{m}, \underline{n}) \neq(\underline{0}, \underline{0})$ or if $m_{i}>1$ for some $i$,

(iii) $\sum_{I \cup J=\{0, \ldots, n\}}|\tilde{\mu}(I, J)| \leqslant 2^{2^{n+1}}$, where, for subsets $I, J \subset\{0, \ldots, n\}, \tilde{\mu}(I, J)$ denotes $\tilde{\mu}\left(m_{0}^{I}, \ldots, m_{n}^{I}, m_{0}^{\prime}{ }^{J}, \ldots, m_{n}^{\prime}\right)$ with $m_{i}^{I}=1$ if $i \in I$ and $m_{i}^{I}=0$ otherwise and $m_{i}{ }^{J}=1$ if $i \in J$ and $m_{i}^{\prime}=0$ otherwise.

Proof. (i) and (ii) follow directly from the definition of $\mu$ immediately above. From the same definition, it follows, if $I \cup J=\{0, \ldots, n\}$, and denoting by $T$ a finite set of subsets $I \subset\{0, \ldots, n\}$, that

$\tilde{\mu}(I, J)=\sum_{m}(-1)^{m} \#\{$ sets $T$ of cardinality $m$

$$
\text { such that } \left.I=\cup_{K \in T} K \text { and } J=\cup_{K \in T} K^{c}\right\} \text {. }
$$

If we sum over all possible $I$ and $J$ such that $I \cup J=\{0, \ldots, n\}$, we get (iii).

Consider now $N_{(\underline{e}, \underline{f})}(B)$ for a couple $(\underline{e}, \underline{f})$ for which $\mu(\underline{e}, \underline{f}) \neq 0$ and

$$
\operatorname{gcd}\left(e_{i} f_{i}, i=0, \ldots, n\right)=e,
$$

i.e., a subset with nontrivial contribution to $\# M(B)$ (recall (25)). Since $\# N_{(e, f)}(B)=$ $\# M_{e^{2} f^{3}, 0}(B)$, choosing $\mu_{i}^{\prime}\left(y_{i}\right)=\mu^{2}\left(f_{i}\left|y_{i}\right|\right)$ (where $\left.e^{2} f^{3}=\left(e_{0}^{2} f_{0}^{3}, \ldots, e_{n}^{2} \bar{f}_{n}^{3}\right)\right)$, we know by Theorem 4.1 that

$$
\# N_{(\underline{e}, \underline{f})}(B)=C_{\underline{e^{2} f^{3}, 0}} \cdot B^{(n-1) / 2}+O\left(B^{(n-1) / 2-\delta}\right) .
$$


Since $e$ divides $e_{i} f_{i}$, we can write $e_{i}^{2} f_{i}^{3}=v_{i} e^{2}$ for some $v_{i} \in \mathbb{N}$ for each $i$ in $\{0, \ldots, n\}$. Making the substitutions $x_{i}^{\prime}=x_{i} / e_{i}$ and $y_{i}^{\prime}=y_{i} / f_{i}$, we see that $N_{(\underline{e}, \underline{f})}(B)$ corresponds to the set

$$
\left\{\left(\underline{x}^{\prime}, \underline{y}^{\prime}\right) \in \mathbb{Z}_{0}^{2 n+2}: \sum_{i=0}^{n} v_{i} x_{i}^{\prime 2} y_{i}^{\prime 3}=0, \max _{0 \leqslant i \leqslant n}\left|v_{i} x_{i}^{\prime 2} y_{i}^{\prime 3}\right| \leqslant \frac{B}{e^{2}} \text { and } \prod_{i=0}^{n} \mu^{2}\left(f_{i}\left|y_{i}^{\prime}\right|\right)=1\right\},
$$

where we eliminated $e^{2}$ in the equation, and hence $\# N_{(\underline{e}, \underline{f})}(B)=\# M_{\underline{v}, 0}\left(B / e^{2}\right)$. Letting $B$ go to infinity, this implies that the main terms in the asymptotic formulas of $\# N_{\underline{e}, \underline{f}}(B)$ and $\# M_{\underline{v}, 0}\left(B / e^{2}\right)$ are equal, and in particular that

$$
\# N_{(\underline{e}, \underline{f})}(B)-C_{\underline{e^{2} f^{3}}, 0} \cdot B^{(n-1) / 2}=O\left(\frac{B^{(n-1) / 2-\delta}}{e^{n-1-2 \delta}}\right) .
$$

Notice we also obtain (recall Remark 4.2) that

$$
\# N_{(\underline{e}, \underline{f})}(B) \leqslant C \cdot \frac{B^{(n-1) / 2}}{e^{n-1}} \quad \text { and } \quad C_{\underline{e}^{2} f^{3}, 0} \leqslant \frac{C}{e^{n-1}} .
$$

From these results, we can now prove:

Lemma 4.5. The series $D=\sum_{e=1}^{\infty} \sum_{\substack{(e, f) \in \mathbb{N}^{2 n+2} \\ \operatorname{gcd}\left(e_{i} f_{i}, i=0, \ldots, n\right)=e}} \mu(\underline{e}, \underline{f}) \cdot C_{\underline{e^{2} f^{3}}, 0}$ converges.

Proof. Substituting (27) into the definition of $D$ and using the properties of $\mu$ in Lemma 4.4, we get

$$
\begin{aligned}
|D| & \ll \sum_{e=1}^{\infty} \sum_{\substack{(\underline{e}, \underline{f}) \in \mathbb{N}^{2 n+2} \\
\operatorname{gcd}\left(e_{i} f_{i}, i=0, \ldots, n\right)=e}} \frac{|\mu(\underline{e}, \underline{f})|}{e^{n-1}} \\
& \leqslant \prod_{p} \sum_{k=0}^{2} \sum_{\substack{\left.v_{p}(\underline{e}), v_{p}(f)\right) \in \mathbb{N}^{2 n+2} \\
\min _{i}\left\{v_{p}\left(e_{i}\right)+v_{p}\left(f_{i}\right)\right\}=k}} \frac{\left|\mu_{p}\left(v_{p}(\underline{e}), v_{p}(\underline{f})\right)\right|}{p^{k(n-1)}} \leqslant \prod_{p}\left(1+2 \frac{2^{2^{n+1}}}{p^{n-1}}\right),
\end{aligned}
$$

which converges since $n \geqslant 4$.

Proof of Theorem 4.3. From the definition of $D$ and (26), it follows that

$$
\left|\# M(B)-D \cdot B^{(n-1) / 2}\right| \ll \sum_{e=1}^{\infty} \sum_{\substack{(e, f) \in \mathbb{N}^{2 n+2} \\ \operatorname{gcd}\left(e_{i} f_{i}, i=0, \ldots, n\right)=e}}|\mu(\underline{e}, \underline{f})| \cdot \frac{B^{(n-1) / 2-\delta}}{e^{(n-1)-2 \delta}} .
$$

Following the same reasoning as in Lemma 4.5, we then get

$$
\left|\# M(B)-D \cdot B^{(n-1) / 2}\right| \ll B^{(n-1) / 2-\delta} \cdot \prod_{p}\left(1+2 \frac{2^{2^{n+1}}}{p^{n-1-2 \delta}}\right),
$$


where the product converges for $\delta>0$ small enough since $n \geqslant 4$. This proves the theorem.

\section{Rational points on the orbifold $\left(\mathbb{P}^{n-1}, \Delta\right)$}

We can now prove our main theorem.

Theorem 5.1. For $n \geqslant 4$, there exists a $\delta>0$ such that

$$
\#\left(\mathbb{P}^{n-1}, \Delta\right)(\mathbb{Q})_{\leqslant B}=C \cdot B^{(n-1) / 2}+O\left(B^{(n-1) / 2-\delta}\right) .
$$

Here,

$$
C=\frac{1}{2^{n+1}} \sum_{e=1}^{\infty} \sum_{\substack{(\underline{e}, \underline{f}) \in \mathbb{N}^{2 n+2} \\ \operatorname{gcd}\left(e_{i} f_{i}, i=0, \ldots, n\right)=e}} \mu(\underline{e}, \underline{f}) \sum_{\substack{y \in \mathbb{Z}_{0}^{n+1} /\{ \pm 1\} \\ f_{i} y_{i} \text { squarefree }}} \frac{2^{n+1} \mathfrak{S}_{\underline{y}, e^{2} f^{3}, 0} \mathfrak{I}_{\underline{\varepsilon}}}{\prod_{i=0}^{n}\left(e_{i}^{2} f_{i}^{3}\left|y_{i}^{3}\right|\right)^{1 / 2}},
$$

with $\mathfrak{S}_{y, \underline{a}, t}, \mathfrak{I}_{\underline{\varepsilon}}$ and the function $\mu$ as defined before. (By $\underline{y} \in \mathbb{Z}_{0}^{n+1} /\{ \pm 1\}$, we denote the $(n+1)$-tuples $\left(y_{0}, \ldots, y_{n}\right) \in \mathbb{Z}_{0}^{n+1}$, defined up to sign as an $(n+1)$-tuple. $)$

Proof. The connection between $\left(\mathbb{P}^{n-1}, \Delta\right)(\mathbb{Q})_{\leqslant B}^{+}$and the set $M(B)$ given by (1), together with Theorem 4.3 , implies that the theorem holds for $\#\left(\mathbb{P}^{n-1}, \Delta\right)(\mathbb{Q})_{\leqslant B}^{+}$. It remains to prove that, for $n \geqslant 4$, the set of points $\left(a_{0}: \cdots: a_{n}\right) \in\left(\mathbb{P}^{n-1}, \Delta\right)(\mathbb{Q})_{\leqslant B}$ with at least one zero coordinate (whose cardinality is $\ll \#\left(\mathbb{P}^{n-2}, \Delta\right)(\mathbb{Q}) \leqslant B$ ), is asymptotically negligible compared to $\left(\mathbb{P}^{n-1}, \Delta\right)(\mathbb{Q})_{\leqslant B}^{+}$.

We will verify this for $n=4$; by induction, the statement follows for $n>4$.

As mentioned in Remark 3.11, it follows from Lemma 3.10 that

$$
\#\left(\mathbb{P}^{2}, \Delta\right)(\mathbb{Q})_{\leqslant B}^{+} \ll B^{1+\varepsilon} .
$$

Combining this with the trivial upper bound $\#\left(\mathbb{P}^{1}, \Delta\right)(\mathbb{Q})_{\leqslant B} \ll B$, we obtain

$$
\#\left(\mathbb{P}^{2}, \Delta\right)(\mathbb{Q})_{\leqslant B} \ll B^{1+\varepsilon}<B^{3 / 2}
$$

for $\varepsilon>0$ sufficiently small.

Description of the constant. An alternative description of $\left(\mathbb{P}^{n-1}, \Delta\right)(\mathbb{Q})_{\leqslant B}^{+}$can be obtained as follows. Consider $\underline{y} \in \mathbb{Z}_{0}^{n+1} /\{ \pm 1\}$ with each $y_{i}$ squarefree. For such $\underline{y}$, let $Q_{y}$ denote the smooth quadric defined by the homogeneous polynomial $F_{\underline{y}}(\underline{x})=y_{0}^{3} X_{0}^{\overline{2}}+\ldots+y_{n}^{3} X_{n}^{2} \in \mathbb{Z}\left[X_{0}, \ldots, X_{n}\right]$. Furthermore, define the morphism

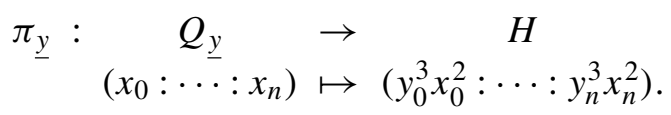

We will consider points $\left(x_{0}: \cdots: x_{n}\right) \in Q_{\underline{y}}(\mathbb{Q})$ with $x_{i} \in \mathbb{Z}$ such that $\prod_{i=0}^{n} x_{i} \neq 0$ and $\operatorname{gcd}\left(x_{0} y_{0}, \ldots, x_{n} y_{n}\right)=1$. We denote this subset of $Q_{\underline{y}}(\mathbb{Q})$ by $Q_{\underline{y}}(\mathbb{Q})^{+}$. This 
set is mapped into $\left(\mathbb{P}^{n-1}, \Delta\right)(\mathbb{Q})^{+}$by $\pi_{\underline{y}}$ and, keeping in mind (1), we have

$$
\left(\mathbb{P}^{n-1}, \Delta\right)(\mathbb{Q})^{+}=\coprod_{\substack{y \in \mathbb{Z}_{0}^{n+1} /\{ \pm 1\} \\ y_{i} \text { squarefree }}} \pi_{\underline{y}}\left(Q_{\underline{y}}(\mathbb{Q})^{+}\right) .
$$

This implies

$$
\#\left(\mathbb{P}^{n-1}, \Delta\right)(\mathbb{Q})_{\leqslant B}^{+}=\frac{1}{2^{n+1}} \sum_{\substack{y \in \mathbb{Z}_{0}^{n+1} /\{ \pm 1\} \\ y_{i} \text { squarefree }}} \#\left\{\left(x_{0}: \cdots: x_{n}\right) \in Q_{\underline{y}}(\mathbb{Q})^{+}: \max _{0 \leqslant i \leqslant n}\left|x_{i}^{2} y_{i}^{3}\right| \leqslant B\right\} .
$$

For a fixed $y$, an asymptotic expression for each of the latter sets using the classical circle method is known (see [Davenport 2005, Chapter 8]) and a Möbius inversion for the $\operatorname{gcd}$ condition $\operatorname{gcd}\left(x_{0} y_{0}, \ldots, x_{n} y_{n}\right)=1$.

Moreover, from Lemma 4.5, it follows that we can change the order of summation for $e$ and $\underline{y}$ in the constant $C$ from Theorem 5.1 and thus, defining

$$
C_{Q_{\underline{y}}}=\sum_{e=1}^{\infty} \sum_{\substack{(\underline{e}, \underline{f}) \in \mathbb{N}^{2 n+2} \\ \operatorname{gcd}\left(e_{i} f_{i}^{-}, i=0, \ldots, n\right)=e \\ f_{i} \mid y_{i}}} \mu(\underline{e}, \underline{f}) \frac{2^{n+1} \mathfrak{S}_{\underline{y}, \underline{e}^{2}, 0 \mathfrak{J}_{\underline{\varepsilon}}}}{\prod_{i=0}^{n}\left(e_{i}\left|y_{i}\right|^{3 / 2}\right)},
$$

we have, for $n \geqslant 4$,

$$
\#\left(\mathbb{P}^{n-1}, \Delta\right)(\mathbb{Q})_{\leqslant B} \sim\left(\frac{1}{2^{n+1}} \sum_{\substack{y \in \mathbb{Z}_{0}^{n+1} /\{ \pm 1\} \\ y_{i} \text { squarefree }}} C_{Q_{\underline{y}}}\right) \cdot B^{(n-1) / 2}
$$

as $B$ goes to infinity.

This constant $C_{Q_{y}}$ can be given a more geometrical interpretation using the adelic space $Q_{y}\left(\mathbb{A}_{\mathbb{Q}}\right)$ of the quadric $Q_{y}$, as explained in [Peyre 1995, §5]. Here, it has been shown that the refined version of the Manin conjecture is compatible with the circle method for smooth quadrics in $\mathbb{P}_{\mathbb{Q}}^{n}$ and moreover, that rational points on smooth quadrics are equidistributed. Considering the Tamagawa measure $\boldsymbol{\omega}_{H_{y}}$ (corresponding to the height function $H_{y}$ defined as $H_{y}(P)=\max _{0 \leqslant i \leqslant n}\left|x_{i}^{2} y_{i}^{3}\right|$ where $\left.P=\left(x_{0}: \cdots: x_{n}\right) \in Q_{y}(\mathbb{Q})\right)$ on $\mathbb{Q}_{y}\left(\mathbb{A}_{\mathbb{Q}}\right)$, the equidistribution of the rational points on $Q_{\underline{y}}$ implies that for every good open subset $W$ (that is, an open subset $W$ for which $\omega_{H_{\underline{y}}}(\partial W)=0$, where $\left.\partial W=\bar{W} \backslash W\right)$ of $Q_{\underline{y}}\left(\mathbb{A}_{\mathbb{Q}}\right)$, we have

$$
\frac{\#\left\{P \in Q_{\underline{y}}(\mathbb{Q})^{+} \cap W \mid H_{\underline{y}}(P) \leqslant B\right\}}{\#\left\{P \in Q_{\underline{y}}(\mathbb{Q})^{+} \mid H_{\underline{y}}(P) \leqslant B\right\}} \rightarrow \frac{\omega_{H_{\underline{y}}}(W)}{\omega_{H_{\underline{y}}}\left(Q_{\underline{y}}\left(\mathbb{A}_{\mathbb{Q}}\right)\right)}
$$

as $B$ goes to infinity. We refer to [Peyre 1995] for more details on this matter. This implies we can obtain a description of the constant $C_{Q_{\underline{y}}}$ in terms of the measure $\boldsymbol{\omega}_{H_{\underline{y}}}$ 
of a certain subset of the adelic space $Q_{\underline{y}}\left(\mathbb{A}_{\mathbb{Q}}\right)$ of the quadric $Q_{\underline{y}}$. More precisely, it follows that

$$
C_{Q_{\underline{y}}}=\omega_{H_{\underline{y}}}\left(Q_{\underline{y}}\left(\mathbb{A}_{\mathbb{Q}}\right)^{\dagger}\right) /(n-1),
$$

where $Q_{\underline{y}}\left(\mathbb{A}_{\mathbb{Q}}\right)^{\dagger}$ denotes the good open subset of $Q_{y}\left(\mathbb{A}_{\mathbb{Q}}\right)$ defined by the gcd condition $\operatorname{gcd}\left(x_{0} y_{0}, \ldots, x_{n} y_{n}\right)=1$ we imposed on $Q_{\underline{y}}(\mathbb{Q})$. (Note that imposing the open condition $\prod_{i=0}^{n} x_{i} \neq 0$ does not change the measure.) We obtain the following corollary.

Corollary 5.2. For $n \geqslant 4$, we have

$$
\#\left(\mathbb{P}^{n-1}, \Delta\right)(\mathbb{Q})_{\leqslant B} \sim\left(\frac{1}{2^{n+1}} \sum_{\substack{y \in \mathbb{Z}_{0}^{n+1} /\{ \pm 1\} \\ y_{i} \text { squarefree }}} C_{Q_{\underline{y}}}\right) \cdot B^{(n-1) / 2}
$$

as $B$ goes to infinity, where $C_{Q_{\underline{y}}}=\omega_{H_{\underline{y}}}\left(Q_{\underline{y}}\left(\mathbb{A}_{\mathbb{Q}}\right)^{\dagger}\right) /(n-1)$.

The adelic space of the orbifold $\left(\mathbb{P}^{n-1}, \Delta\right)$. In order to define the adelic space of the orbifold properly, we first have to explain how we can translate the definition of "squarefulness" to the different completions of $\mathbb{Q}$.

At each finite place $v=p$, a $p$-adic integer $a \in \mathbb{Z}_{p}$ is squareful if $v_{p}(a) \neq 1$. Due to the structure of $\mathbb{Q}_{p}^{\times}$, this means that we can write a squareful $p$-adic integer $a$ uniquely as $x^{2} y^{3}$ with $x \in \mathbb{Z}_{p}^{\times}$and $y \in \mathbb{Z}$ squarefree.

On the other hand, any real number $a \in \mathbb{R}$ can be written as $( \pm 1)^{3} x^{2}$ and ought to be considered as squareful.

Since we identified $\left(\mathbb{P}^{n-1}, \Delta\right)(\mathbb{Q})$ with $\left\{\left(u_{0}: \cdots: u_{n}\right) \in H(\mathbb{Q}): u_{i}\right.$ squareful $\}$ (recall $H \subset \mathbb{P}^{n}$ is the hyperplane defined by $X_{0}+\cdots+X_{n}=0$ ), we have, for each $v \in \operatorname{Val}(\mathbb{Q})$, that

$$
\begin{aligned}
\left(\mathbb{P}^{n-1}, \Delta\right)\left(\mathbb{Q}_{v}\right) & =\left\{\left(u_{0}: \cdots: u_{n}\right) \in H\left(\mathbb{Q}_{v}\right): u_{i} \text { squareful }\right\} \\
& =\left\{\left(x_{0, v}^{2} y_{0}^{3}: \cdots: x_{n, v}^{2} y_{n}^{3}\right) \in H\left(\mathbb{Q}_{v}\right): \underline{y} \in \mathbb{Z}_{0}^{n+1} /\{ \pm 1\}, y_{i} \text { squarefree }\right\} .
\end{aligned}
$$

This implies, recalling the definition of $\pi_{\underline{y}}$ in (28),

$$
\left(\mathbb{P}^{n-1}, \Delta\right)\left(\mathbb{Q}_{v}\right)=\bigcup_{\substack{y \in \mathbb{Z}_{0}^{n+1} /\{ \pm 1\} \\ y_{i} \text { squarefree }}} \pi_{\underline{y}}\left(Q_{\underline{y}}\left(\mathbb{Q}_{v}\right)^{\dagger}\right),
$$

where for a finite place $v=p, Q_{\underline{y}}\left(\mathbb{Q}_{p}\right)^{\dagger}$ is the open subset of $Q_{\underline{y}}\left(\mathbb{Q}_{p}\right)$ defined by the condition $\min _{0 \leqslant i \leqslant n}\left(v_{p}\left(x_{i, p} y_{i}\right)\right)=0$, and where $Q_{y}(\mathbb{R})^{\dagger}=Q_{y}(\mathbb{R})$.

Note that the union considered is not disjoint, but that the image for different $\underline{y}$ and $\underline{y}^{\prime}$ either coincides or is disjoint. Hence, it follows that, at each place $\bar{v} \in \operatorname{Val}(\mathbb{Q}),\left(\mathbb{P}^{n-1}, \Delta\right)\left(\mathbb{Q}_{v}\right)$ can be described as a finite disjoint union of sets $\pi_{\underline{y}}\left(Q_{\underline{y}}\left(\mathbb{Q}_{v}\right)^{\dagger}\right)$ for specified $\underline{y} \in \mathbb{Z}_{0}^{n+1} /\{ \pm 1\}$. 
Definition. We define the adelic space $\left(\mathbb{P}^{n-1}, \Delta\right)\left(\mathbb{A}_{\mathbb{Q}}\right)$ as

$$
\left(\mathbb{P}^{n-1}, \Delta\right)\left(\mathbb{A}_{\mathbb{Q}}\right)=\prod_{v \in \operatorname{Val}(\mathbb{Q})}\left(\mathbb{P}^{n-1}, \Delta\right)\left(\mathbb{Q}_{v}\right) .
$$

Remark 5.3. One may prove that $\left(\mathbb{P}^{n-1}, \Delta\right)(\mathbb{Q})$ is dense in $\left(\mathbb{P}^{n-1}, \Delta\right)\left(\mathbb{A}_{\mathbb{Q}}\right)$. This follows from the fact that weak approximation holds for smooth quadrics.

Distribution of rational points on $\left(\mathbb{P}^{n-1}, \Delta\right)$. We can now consider the probability measure

$$
\mu_{H \leqslant B}^{\left(\mathbb{P}^{n-1}, \Delta\right)}=\frac{1}{\#\left(\mathbb{P}^{n-1}, \Delta\right)(\mathbb{Q})_{\leqslant B}} \sum_{\substack{P \in\left(\mathbb{P}^{n-1}, \Delta\right)(\mathbb{Q}) \\ H(P) \leqslant B}} \delta_{P}
$$

on $\left(\mathbb{P}^{n-1}, \Delta\right)\left(\mathbb{A}_{\mathbb{Q}}\right)$. Here, we will investigate the convergence of $\mu_{H \leqslant B}^{\left(\mathbb{P}^{n-1}, \Delta\right)}$ to a specific measure on the adelic space of the orbifold, which we have yet to define, when $B$ goes to infinity. Keeping in mind the description of $\left(\mathbb{P}^{n-1}, \Delta\right)\left(\mathbb{A}_{\mathbb{Q}}\right)$ we gave above, we can define this measure in the following natural way.

Definition. We define the measure $\boldsymbol{\omega}_{\left(\mathbb{P}^{n-1}, \Delta\right)}$ on $\left(\mathbb{P}^{n-1}, \Delta\right)\left(\mathbb{A}_{\mathbb{Q}}\right)$ as

$$
\boldsymbol{\omega}_{(\mathbb{P} n-1, \Delta)}(U)=\sum_{\substack{y \in \mathbb{Z}_{0}^{n+1} /\{ \pm 1\} \\ y_{i} \text { squarefree }}} \boldsymbol{\omega}_{H_{\underline{y}}}\left(\pi_{\underline{y}}^{-1}(U)\right),
$$

where $U$ is an open subset of $\left(\mathbb{P}^{n-1}, \Delta\right)\left(\mathbb{A}_{\mathbb{Q}}\right)$ (which is equipped with the subspace topology coming from $\left.H\left(\mathbb{A}_{\mathbb{Q}}\right)\right)$ and $\pi_{\underline{y}}: Q_{\underline{y}}\left(\mathbb{A}_{\mathbb{Q}}\right)^{\dagger} \rightarrow\left(\mathbb{P}^{n-1}, \Delta\right)\left(\mathbb{A}_{\mathbb{Q}}\right)$. (Note that the morphisms $\pi_{\underline{y}}$ introduced in $(28)$ define continuous maps $\pi_{\underline{y}}: Q_{\underline{y}}\left(\mathbb{A}_{\mathbb{Q}}\right) \rightarrow$ $H\left(\mathbb{A}_{\mathbb{Q}}\right)$ which map $Q_{\underline{y}}\left(\mathbb{A}_{\mathbb{Q}}\right)^{\dagger}$ into $\left(\mathbb{P}^{n-1}, \Delta\right)\left(\mathbb{A}_{\mathbb{Q}}\right)$.)

Remark 5.4. From this definition of the measure $\omega_{\left(\mathbb{P}^{n-1}, \Delta\right)}$, it follows that its support consists of the (disjoint) union of

$$
\pi_{\underline{y}}\left(Q_{\underline{y}}\left(\mathbb{A}_{\mathbb{Q}}\right)^{\dagger}\right)
$$

for all $\underline{y} \in \mathbb{Z}_{0}^{n+1} /\{ \pm 1\}$ with $y_{i}$ squarefree for each $i \in\{0, \ldots, n\}$. This is a proper subset of $\left(\mathbb{P}^{n-1}, \Delta\right)\left(\mathbb{A}_{\mathbb{Q}}\right)$.

In order to say something about the convergence of $\mu_{H \leqslant B}^{\left(\mathbb{P}^{n-1}, \Delta\right)}$, we first define elementary open subsets of $\left(\mathbb{P}^{n-1}, \Delta\right)\left(\mathbb{A}_{\mathbb{Q}}\right)$.

An elementary open subset $W$ of $H\left(\mathbb{A}_{\mathbb{Q}}\right)$ can be defined as

$$
W=\prod_{v \in \operatorname{Val}(\mathbb{Q})} W_{v},
$$

such that $W_{v} \subset H\left(\mathbb{Q}_{v}\right)$ is defined at finitely many finite places as $W_{p}=\operatorname{red}_{M}^{-1}\left(X_{p}\right)$, where $X_{p} \subset H\left(\mathbb{Z} / p^{M} \mathbb{Z}\right)$ and $\operatorname{red}_{M}: H\left(\mathbb{Q}_{p}\right) \rightarrow H\left(\mathbb{Z} / p^{M} \mathbb{Z}\right) ; W_{p}=H\left(\mathbb{Q}_{p}\right)$ for 
any other finite place. Furthermore, at the infinite place $v=\infty$, we require $W_{\infty}=$ $\bigcap_{i, j}\left(\lambda_{i, j} x_{i}<x_{j}\right) \subset H(\mathbb{R})$ fixing one of the coordinates $x_{i}$ to one. Here, $\lambda_{i, j} \in \mathbb{R}_{>0}$ depending on $i$ and $j$.

To construct elementary open subsets on $\left(\mathbb{P}^{n-1}, \Delta\right)\left(\mathbb{A}_{\mathbb{Q}}\right)$, we can take the intersection with elementary open subsets of $H\left(\mathbb{A}_{\mathbb{Q}}\right)$.

We will now prove the following theorem.

Theorem 5.5. For every elementary open subset $U$ of $\left(\mathbb{P}^{n-1}, \Delta\right)\left(\mathbb{A}_{\mathbb{Q}}\right)$, we have

$$
\mu_{H \leqslant B}^{\left(\mathbb{P}^{n-1}, \Delta\right)}(U) \rightarrow \frac{\omega_{\left(\mathbb{P}^{n-1}, \Delta\right)}(U)}{\boldsymbol{\omega}_{\left(\mathbb{P}^{n-1}, \Delta\right)}\left(\left(\mathbb{P}^{n-1}, \Delta\right)\left(\mathbb{A}_{\mathbb{Q}}\right)\right)}
$$

as $B$ goes to infinity.

Proof. Straightforward calculations show that for each admitted $\underline{y}$, the inverse image $\pi_{\underline{y}}^{-1}(U)$ of an elementary open subset $U$ of $\left(\mathbb{P}^{n-1}, \Delta\right)\left(\mathbb{A}_{\mathbb{Q}}\right)$ defines a good open subset of $Q_{\underline{y}}\left(\mathbb{A}_{\mathbb{Q}}\right)^{\dagger}$.

Now let $U$ be an elementary open subset of $\left(\mathbb{P}^{n-1}, \Delta\right)\left(\mathbb{A}_{\mathbb{Q}}\right)$. Recalling (33), the partition of $\left(\mathbb{P}^{n-1}, \Delta\right)(\mathbb{Q})^{+}$in $(29)$, and Theorem 5.1 , we get

$$
\begin{aligned}
\mu_{H \leqslant B}^{\left(\mathbb{P}^{n-1}, \Delta\right)}(U) & =\frac{\#\left\{\left(u_{0}: \cdots: u_{n}\right) \in\left(\mathbb{P}^{n-1}, \Delta\right)(\mathbb{Q}) \cap U: \max _{0 \leqslant i \leqslant n}\left|u_{i}\right| \leqslant B\right\}}{\#(\mathbb{P} n-1, \Delta)(\mathbb{Q})_{B} \leqslant B} \\
& \sim \frac{\sum_{\underline{y}} \#\left\{\left(x_{0}: \cdots: x_{n}\right) \in Q_{\underline{y}}(\mathbb{Q})^{+} \cap \pi_{\underline{y}}^{-1}(U): \max _{0 \leqslant i \leqslant n}\left|y_{i}^{3} x_{i}^{2}\right| \leqslant B\right\}}{\sum_{\underline{y}} \#\left\{\left(x_{0}: \cdots: x_{n}\right) \in Q_{\underline{y}}(\mathbb{Q})^{+}: \max _{0 \leqslant i \leqslant n}\left|y_{i}^{3} x_{i}^{2}\right| \leqslant B\right\}} .
\end{aligned}
$$

(Here, we used the abbreviated notation $\sum_{y}$ to sum over all admitted $\underline{y} \in \mathbb{Z}_{0}^{n+1}$.)

Combining the fact that rational points on smooth quadrics are equidistributed, the definition of the measure in (34), and Theorem 5.1 enables us to complete the proof.

\section{Acknowledgments}

I would like to express my gratitude to my advisor professor Emmanuel Peyre for the many helpful conversations concerning these subjects, and to Number Theory section of the department of Mathematics of the University of Bristol (in particular professor Tim Browning and professor Trevor Wooley) for the useful tips concerning the circle method. Also, I would like to thank the reviewers for some interesting comments which led to substantial improvements of the result and the presentation.

\section{References}

[Abramovich 2009] D. Abramovich, "Birational geometry for number theorists", pp. 335-373 in Arithmetic geometry (Göttingen, 2006), edited by H. Darmon et al., Clay Math. Proc. 8, Amer. Math. Soc., Providence, RI, 2009. MR 2010m:14021 Zbl 1192.14013 
[Berndt et al. 1998] B. C. Berndt, R. J. Evans, and K. S. Williams, Gauss and Jacobi sums, Wiley, New York, 1998. MR 99d:11092 Zbl 0906.11001

[Campana 2005] F. Campana, "Fibres multiples sur les surfaces: aspects geométriques, hyperboliques et arithmétiques", Manuscripta Math. 117:4 (2005), 429-461. MR 2006e:14013 Zbl 1129.14051

[Davenport 2005] H. Davenport, Analytic methods for Diophantine equations and Diophantine inequalities, 2nd ed., Cambridge University Press, 2005. MR 2006a:11129 Zbl 1125.11018

[Peyre 1995] E. Peyre, "Hauteurs et mesures de Tamagawa sur les variétés de Fano", Duke Math. J. 79:1 (1995), 101-218. MR 96h:11062 Zbl 0901.14025

[Poonen 2006] B. Poonen, "The projective line minus three fractional points", 2006, available at http://www-math.mit.edu/ poonen/slides/campana_s.pdf.

[Schmidt 1984] W. M. Schmidt, Analytische Methoden für Diophantische Gleichungen. Einführende Vorlesungen, DMV Seminar 5, Birkhäuser, Basel, 1984. MR 86f:11024 Zbl 0573.10013

[Vaughan 1997] R. C. Vaughan, The Hardy-Littlewood method, 2nd ed., Cambridge Tracts in Mathematics 125, Cambridge University Press, 1997. MR 98a:11133 Zbl 0868.11046

Communicated by Bjorn Poonen

Received 2010-12-03 Revised 2011-06-17 Accepted 2011-07-19

karl.vanvalckenborgh@kbc.be Department of Mathematics, Katholieke Universiteit Leuven, Celestijnenlaan 200B, 3001 Leuven, Belgium 


\section{Algebra \& Number Theory}

msp.berkeley.edu/ant

\section{EDITORS}

MANAGING EDITOR

Bjorn Poonen

Massachusetts Institute of Technology

Cambridge, USA

\author{
EDITORIAL BOARD CHAIR \\ David Eisenbud \\ University of California \\ Berkeley, USA
}

\section{BOARD OF EDITORS}

Georgia Benkart

Dave Benson

Richard E. Borcherds

John H. Coates

J-L. Colliot-Thélène

Brian D. Conrad

Hélène Esnault

Hubert Flenner

Edward Frenkel

Andrew Granville

Joseph Gubeladze

Ehud Hrushovski

Craig Huneke

Mikhail Kapranov

Yujiro Kawamata

János Kollár

Yuri Manin

Barry Mazur

Philippe Michel
University of Wisconsin, Madison, USA

University of Aberdeen, Scotland

University of California, Berkeley, USA

University of Cambridge, UK

CNRS, Université Paris-Sud, France

University of Michigan, USA

Universität Duisburg-Essen, Germany

Ruhr-Universität, Germany

University of California, Berkeley, USA

Université de Montréal, Canada

San Francisco State University, USA

Hebrew University, Israel

University of Kansas, USA

Yale University, USA

University of Tokyo, Japan

Princeton University, USA

Northwestern University, USA

Harvard University, USA

École Polytechnique Fédérale de Lausanne
Susan Montgomery

Shigefumi Mori

Raman Parimala

Jonathan Pila

Victor Reiner

Karl Rubin

Peter Sarnak

Joseph H. Silverman

Michael Singer

Vasudevan Srinivas

J. Toby Stafford

Bernd Sturmfels

Richard Taylor

Ravi Vakil

Michel van den Bergh

Marie-France Vignéras

Kei-Ichi Watanabe

Andrei Zelevinsky

Efim Zelmanov
University of Southern California, USA

RIMS, Kyoto University, Japan

Emory University, USA

University of Oxford, UK

University of Minnesota, USA

University of California, Irvine, USA

Princeton University, USA

Brown University, USA

North Carolina State University, USA

Tata Inst. of Fund. Research, India

University of Michigan, USA

University of California, Berkeley, USA

Harvard University, USA

Stanford University, USA

Hasselt University, Belgium

Université Paris VII, France

Nihon University, Japan

Northeastern University, USA

University of California, San Diego, USA

\section{PRODUCTION}

contact@msp.org

Silvio Levy, Scientific Editor

See inside back cover or www.jant.org for submission instructions.

The subscription price for 2012 is US \$175/year for the electronic version, and \$275/year (+ \$40 shipping outside the US) for print and electronic. Subscriptions, requests for back issues from the last three years and changes of subscribers address should be sent to Mathematical Sciences Publishers, Department of Mathematics, University of California, Berkeley, CA 94720-3840, USA.

Algebra \& Number Theory (ISSN 1937-0652) at Mathematical Sciences Publishers, Department of Mathematics, University of California, Berkeley, CA 94720-3840 is published continuously online. Periodical rate postage paid at Berkeley, CA 94704, and additional mailing offices.

ANT peer review and production are managed by EditFLOW ${ }^{\circledR}$ from Mathematical Sciences Publishers.

PUBLISHED BY

mathematical sciences publishers

http://msp.org/

A NON-PROFIT CORPORATION

Typeset in IATEX

Copyright $@ 2012$ by Mathematical Sciences Publishers 


\section{Algebra \& Number Theory}

Volume $6 \quad$ No. $5 \quad 2012$

Fields of moduli of three-point $G$-covers with cyclic $p$-Sylow, I

ANDREW OBUS

Toroidal compactifications of PEL-type Kuga families

KAI-WEN LAN

Idempotents in representation rings of quivers

RYAN KINSER and RALF SCHIFFLER

Cox rings and pseudoeffective cones of projectivized toric vector bundles

José González, Milena Hering, Sam Payne and Hendrik SÜss

Squareful numbers in hyperplanes

KARL VAN VALCKENBORGH

A denominator identity for affine Lie superalgebras with zero dual Coxeter number MARIA GORELIK and SHIFRA REIF 